\title{
On the Interaction between Wind Stress and Waves: Wave Growth and Statistical Properties of Large Waves
}

\author{
J. H. LEE AND J. P. MONTY \\ University of Melbourne, Parkville, Victoria, Australia
}

(Manuscript received 8 May 2019, in final form 25 October 2019)

\begin{abstract}
Statistical properties and development of wave fields with different wind forcings are investigated through parametric laboratory experiments. Thirty different, random sea states simulated using a JONSWAP spectrum are mechanically generated in deep-water conditions. Each of the random simulated sea states is exactly repeated but subjected to a range of different wind speeds to study the interaction between wind stress and the existing random sea state waves, especially the isolated effect of the wind stress on the largest waves. Wave crest distributions are sensitive to the wind at the extreme end such that there is an observed deviation from second-order theory for the largest (lowest probability) waves at high wind speed. Because the local wave steepness increases with wind speed, eventually reaching a breaking point, the growth of extreme waves (relative to the significant wave height) due to wind stress is shown to be limited by wave breaking. Even when large waves are breaking, the data reveal that amplitude modulation of wave groups is enhanced substantially as the wind speed increases due to the difference in growth rates between the highest and the lowest wave crests in a wave group. However, there is no evidence of an increase in modulation instability with the wind speed, suggesting that the wind-wave interaction under strong wind forcing dominates the wave growth mechanism over nonlinear wave interactions in a broadband wave field.
\end{abstract}

\section{Introduction}

The evolution of large-amplitude surface gravity waves is critical to many metocean processes and so to many aspects of geophysical modeling and prediction. From an engineering standpoint, large waves need to be predicted and/or considered at the design stage for naval architecture, oil and gas, shipping, and coastal engineering industries. For example, knowing or forecasting the occurrence of extreme waves is critical to the estimation of wave forces and prediction of structural response in the design of offshore platforms. Following the general acceptance of rogue waves as physical phenomena (Dysthe et al. 2008), the past two decades saw a large increase in the amount of research into the most extreme (height) waves in a given sea state. Studies have proposed a range of physical formation mechanisms such as linear focusing of different waves (Kharif and Pelinovsky 2003), interactions between wave and current (White and Fornberg 1998), and nonlinear interaction in wave fields (Fedele et al. 2016) due to dispersive focusing of second-order nonresonant waves

Corresponding author: J. H. Lee, jung.lee@unimelb.edu.au
(Fedele and Tayfun 2009) or third-order wave interaction associated with the modulational instability (Benjamin and Feir 1967; Zakharov 1968; Janssen 2003; Chabchoub et al. 2011).

While it is evident from the literature that a range of nonlinear mechanisms are at play in large wave growth, the role of wind (if any) in nonlinear focusing has received far less attention to date. Indeed it is unclear whether the nonlinear interactions are suppressed or amplified by the wind. Experiments on monochromatic wave trains have shown that the growth in naturally developing sideband energy, characteristic of modulation instability, is suppressed (Bliven et al. 1986; Li et al. 1988) or unchanged (Waseda and Tulin 1999). Wind forcing of more complex wave trains has shown that the amplitude of extreme waves is increased by wind using both numerical and experimental approaches (Kharif et al. 2008; Chabchoub et al. 2013). In terms of nonlinear interactions, modulation instability is still an active and observable contributor to wave-wave dynamics even at high wind speed (Chabchoub et al. 2013). Brunetti and Kasparian (2014) show analytically that by adding a wind forcing term to the nonlinear Schrödinger (NLS) equations, there may even be an enhancement of the 

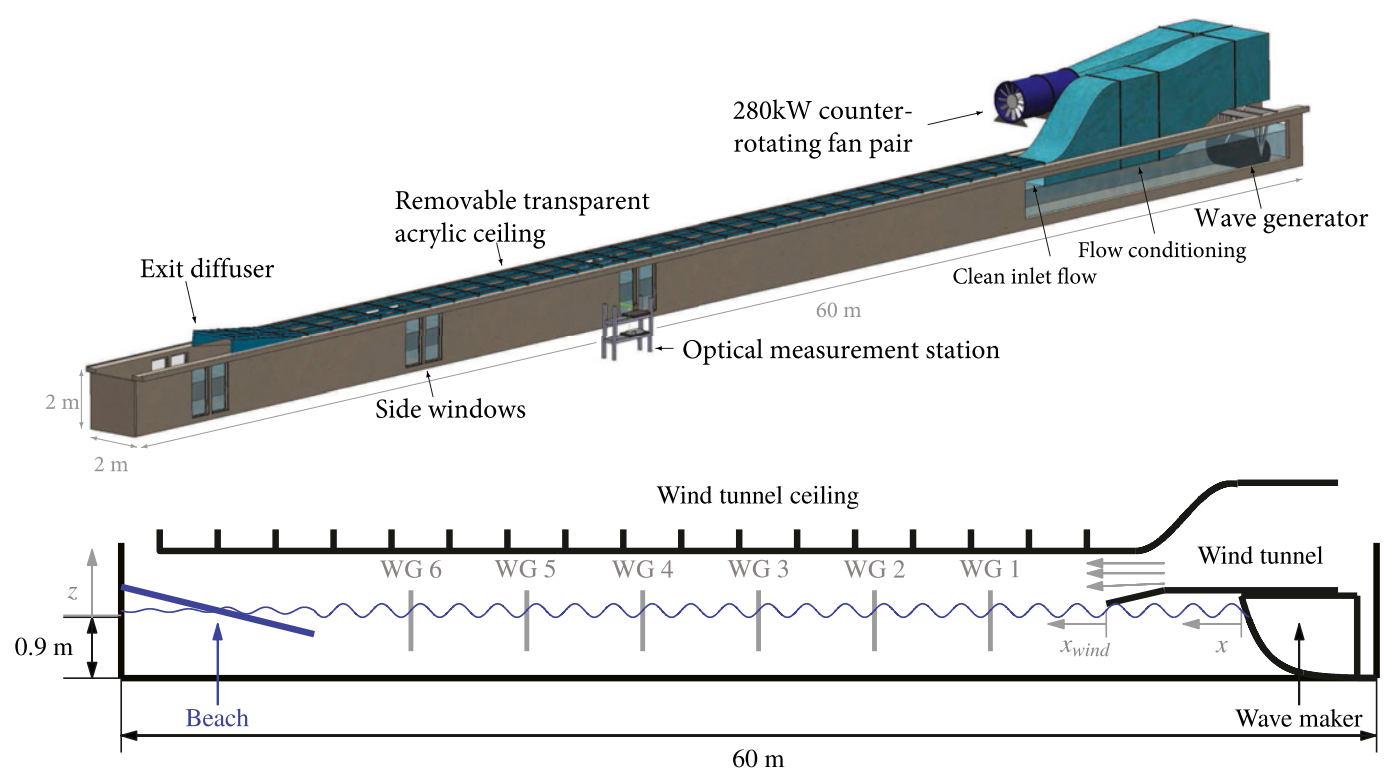

FIG. 1. Schematic of wave tank and experimental setup. The upper figure is drawn to scale, while the lower figure is not to scale.

modulational instability due to the wind, though it is emphasized that these authors also highlight the need for experiments or observations to validate their results.

In practice, engineers rely on probability distributions of wave heights for design and safety procedures. In particular, the likelihood of encountering very large waves is needed. Previous studies have attempted to predict the statistical properties of surface gravity waves employing both first- and second-order theories. In the linear theory, under the narrowband spectrum condition, free surface elevation is described by a Gaussian distribution and wave crests follow the Rayleigh distribution. Longuet-Higgins (1963) derived a general solution for the surface elevation up to second order using the Stokes expansion to take the nonlinearity of water waves into account for the deep-water condition. Later, Tayfun (1980) used the Stokes model and the extra assumptions that waves are unidirectional and narrowbanded to derive a correction to the Rayleigh distribution for wave crests, especially for the extreme cases. Unsurprisingly, there is limited data on extreme waves in different sea states with which to validate corrections. Dysthe et al. (2003) and Socquet-Juglard et al. (2005) performed numerical simulations to investigate the stability of narrowbanded waves and the effect of the spectral evolution on the probability distributions of surface elevation and crest height. They report agreement with the second-order theory proposed by Tayfun (1980) for short-crested waves, but their simulations showed a deviation for large waves. These observations are consistent with the experimental study performed by Onorato et al. (2006). A popular second-order model that includes the combined effects of wave steepness, directionality and water depth was proposed by Forristall (2000). The resulting wave crest probability distribution function agrees well with data from field experiments. The model also included a parameterization obtained from simulations using a set of JONSWAP spectra to compute wave crest exceedance probabilities for engineering applications.

Unfortunately, there are few studies that have systematically studied practically important statistics of evolving complex wave trains. There are some notable works, for example, Waseda (2006), Denissenko et al. (2007), Onorato et al. (2009), and Latheef and Swan (2013), that have investigated the probability distributions of wave crests and the effects of the wave directionality for such wave fields. These studies considered an extensive range of wave directional distributions with mechanically generated wave fields. In general, the conclusions were that for long-crested, narrowbanded, and steep waves, the second-order models of Tayfun (1980) and Forristall (2000) underestimate the exceedance probabilities of large waves, however, the deviation decreases slightly when the directionality of wave fields is included (becoming short-crested waves). However, none of the above studies includes the influence of wind and associated interaction of wind forcing with waves; the effect of wind on the statistical properties of the random sea state waves due to the wind-wave interaction remains largely unknown. 


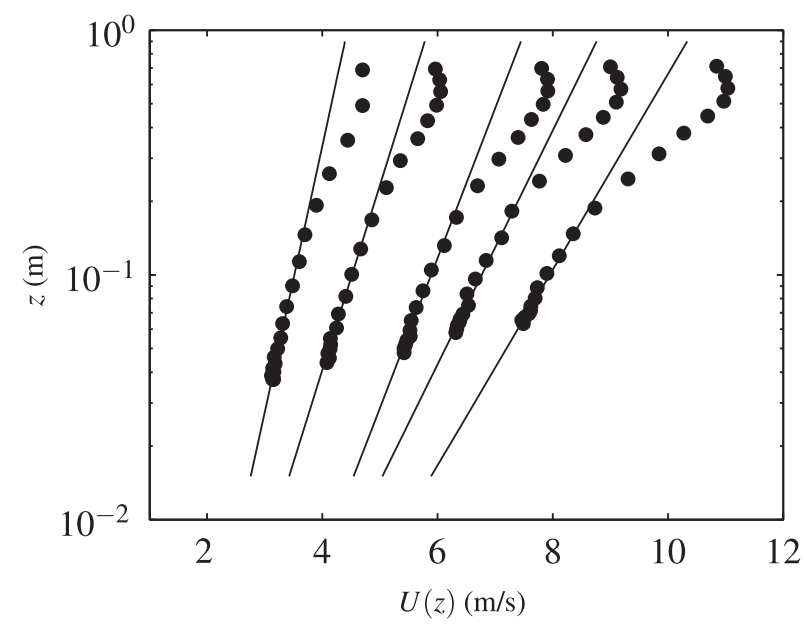

FIG. 2. Wind profiles $U(z)$ over the water surface with different wind speeds measured at $x=28 \mathrm{~m}$ from the wave maker, where $x$ is distance from the wave maker (wind fetch is approximately $21 \mathrm{~m}$ ). The circles are Pitot-static tube data, and the solid line indicates the logarithmic fit (1).

In this paper we report the results of a carefully designed and controlled laboratory experiment to study the combined effects of random sea state and wind forcing, aiming to provide insights into the open question of the role of wind in nonlinear interactions between waves and the probability of large (extreme) waves.

\section{Experimental setup}

Experiments are performed in the Extreme AirSea Interaction (EASI) facility located in the Michell Hydrodynamics Laboratory at the University of Melbourne. A schematic of the facility is shown in Fig. 1. This facility consists of an open-return blower wind tunnel built over a wave tank with a working section of $60 \mathrm{~m} \times 1.8 \mathrm{~m} \times 2 \mathrm{~m}$ (length $\times$ width $\times$ height) and the maximum water depth is $1.1 \mathrm{~m}(0.9-\mathrm{m}$ depth is used in this study). The wave tank is equipped with a computercontrolled oscillating cam-type mechanical wave maker that can generate waves with predefined wave forms and a minimum-reflection beach that dissipates incoming wave energy (Lee et al. 2017). The wind tunnel is capable of generating free-stream velocities up to $35 \mathrm{~m} \mathrm{~s}^{-1}$. The flow generated by a pair of $280-\mathrm{kW}$ counter rotating fans is treated to reduce turbulence by first passing through a honeycomb sheet (honeycomb cells with $6-\mathrm{mm}$ diameter and $80-\mathrm{mm}$ length) for flow straightening. The flow continues through four stainless steel fine-mesh screens to dissipate smaller-scale turbulence, before passing through a two-dimensional contraction with an area ratio of 7:1 to stretch the remaining turbulence and accelerate the flow to the working speed. The flow exits the contraction over an adjustable shallow-angled flap to enter the water surface practically parallel to the water (avoiding air impact on the surface) with very low turbulence intensity of nominally $\pm 0.5 \%$. The airflow then develops with a fetch of over $32 \mathrm{~m}$, resulting in a high Reynolds number boundary layer over the water surface. Throughout this paper, $x$ and $z$ will be used to denote the streamwise fetch length from the wave maker (wave-propagation direction) and vertical height referenced to the mean water depth $(\approx 0.9 \mathrm{~m})$, respectively. Overbars indicate time-averaged values.

All velocity profile measurements for different wind speeds are made using a vertically traversing Pitot-static tube located $28 \mathrm{~m}$ from the wave maker and at the crossstream center plane of the tunnel. Figure 2 shows examples of the measured velocity profiles for various wind speeds. The velocity at $10-\mathrm{m}$ height $U_{10}$ is extrapolated using the logarithmic fit in Eq. (1) to the mean velocity profiles with the $\log$ law constant $\kappa=0.41$. The range of free-stream velocity $U_{\infty}$ tested in the current study is $0<U_{\infty}<15 \mathrm{~m} \mathrm{~s}^{-1}$ and corresponding $U_{10}$ values are listed in Table 1:

$$
U(z)=\frac{U_{*}}{\kappa} \ln \left(\frac{z}{z_{0}}\right) .
$$

All wave height measurements are made using Akamina AWP-300-3 capacitance-type wave height gauges with a sampling rate of $50 \mathrm{~Hz}$. A total of six wave gauges are placed along the centerline of the tunnel at a distance of $8,13,18,23,28$, and $31 \mathrm{~m}$ from the wave maker. The wind tunnel outlet is located $7 \mathrm{~m}$ downstream of the wave maker such that the first wave gauge is approximately $1 \mathrm{~m}$ downstream of the wind tunnel exit. The streamwise fetch length from the wind tunnel outlet is denoted by $x_{\text {wind }}$ $(=x-7 \mathrm{~m})$. Figure 1 shows a schematic of the wave gauge setup. Placing the wave gauges at different fetch lengths allow us to observe the spatial evolution of wave height with different wind inputs.

TABLE 1. Experimental parameters measured at $x / L=17.9$ (or $x_{\text {wind }} / L=13.5$ ) for the JONSWAP spectrum with wind experiments; $T_{p}=1 \mathrm{~s}, \alpha=0.02, \gamma=3.3$, and $L=1.56 \mathrm{~m}$ for all cases.

\begin{tabular}{cccccc}
\hline \hline Case & $U_{\infty}\left(\mathrm{m} \mathrm{s}^{-1}\right)$ & $U_{10}\left(\mathrm{~m} \mathrm{~s}^{-1}\right)$ & $U_{*}\left(\mathrm{~m} \mathrm{~s}^{-1}\right)$ & $H_{s}(\mathrm{~mm})$ & $k_{p} H_{s} / 2$ \\
\hline 1 & 0 & 0 & 0 & 57 & 0.12 \\
2 & 4.8 & 5.5 & 0.17 & 60 & 0.121 \\
3 & 6.0 & 7.0 & 0.22 & 62 & 0.125 \\
4 & 7.3 & 8.5 & 0.28 & 66 & 0.132 \\
5 & 8.6 & 10.0 & 0.33 & 73 & 0.147 \\
6 & 9.8 & 11.5 & 0.39 & 80 & 0.162 \\
7 & 11.1 & 13.0 & 0.44 & 90 & 0.183 \\
8 & 12.3 & 14.6 & 0.50 & 102 & 0.201 \\
9 & 13.6 & 16.1 & 0.56 & 117 & 0.237 \\
\hline
\end{tabular}



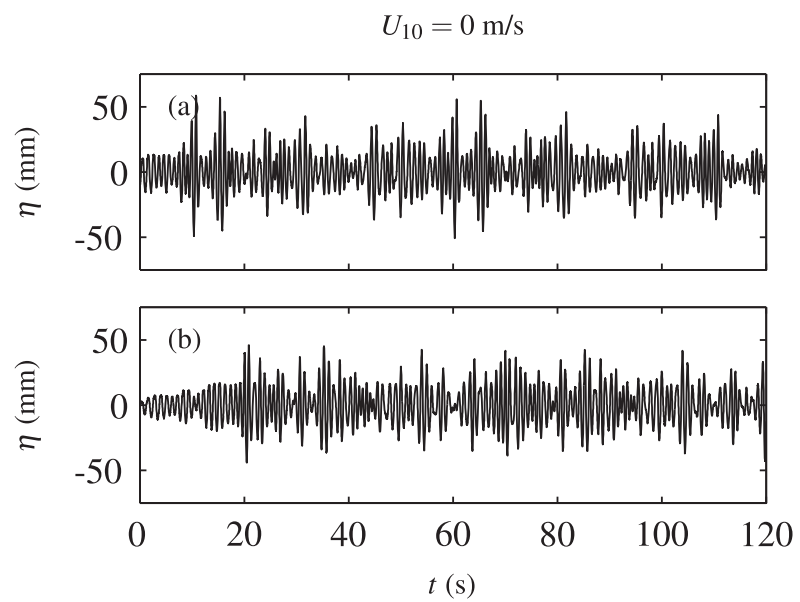

FIG. 3. Instantaneous water surface elevation without wind (case 1) at two different fetch lengths from the wave maker: (a) $x_{\text {wind }} / L=0.65$ and (b) $x_{\text {wind }} / L=13.5$.

To simulate sea states, the JONSWAP spectrum with random phase has been employed to generate initial wave fields (Komen et al. 1994; Onorato et al. 2006):

$$
S(f)=\frac{\alpha g^{2}}{(2 \pi)^{4} f^{5}} \exp \left[-\frac{5}{4}\left(\frac{f}{f_{0}}\right)^{-4}\right] \gamma^{r},
$$

where

$$
r=\exp \left[-\frac{\left(f-f_{0}\right)^{2}}{2 \sigma_{0}^{2} f_{0}^{2}}\right],
$$

where $\alpha$ is the Phillips constant, $g$ is the gravitational acceleration, $f$ is the wave frequency, $f_{0}$ is the frequency at the spectral peak, $\gamma$ is the peak enhancement parameter, $\sigma_{0}=0.07$ when $f \leq f_{0}$, and $\sigma_{0}=0.09$ when $f>f_{0}$. Throughout this study, we have used $f_{0}=1 \mathrm{~Hz}$ which is equivalent to $T_{p}=1 \mathrm{~s}$ where $T_{p}$ is peak period and the input JONSWAP spectrum is truncated at $f>2 \mathrm{~Hz}$ due to the limited output frequency range of the wave maker. The peak period of $1 \mathrm{~s}$ corresponds to a wavelength $L$ of $1.56 \mathrm{~m}$ assuming linear dispersion of the dominant waves. Parameters $\alpha=0.02$ and $\gamma=3.3$ are chosen to generate an input JONSWAP spectrum similar to Onorato et al. (2009). The experimental parameters for the measurements with different wind inputs considered in the current study are provided in Table 1 including the wave steepness $k_{p} H_{s} / 2$, where $k_{p}$ is the wavenumber corresponding to the peak wave frequency and $H_{s}$ is the significant wave height. It is emphasized that predefined wave forms generated using the JONSWAP spectrum are mechanically forced by the computer controlled mechanical wave generator, while a range of different wind

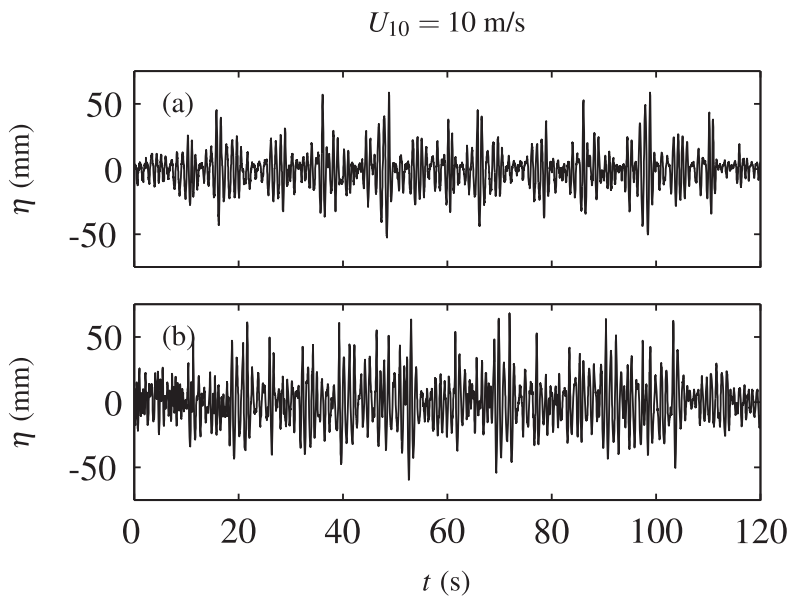

FIG. 4. Instantaneous water surface elevation with wind (case 5) at two different fetch lengths from the wave maker: (a) $x_{\text {wind }} / L=$ 0.65 and (b) $x_{\text {wind }} / L=13.5$.

forces is imposed over the mechanical waves using the fully automated wind tunnel. 30 runs of random waves with 120-s duration are generated for each case (listed in Table 1) to ensure converged wave statistics. A subtle, yet crucial, detail to note is that the exact same set of 30 random wave forms is mechanically generated in each case, such that the only difference between the cases is the input wind speed.

\section{Results and discussion}

Figures 3 and 4 show sample surface elevations $\eta$ having the same mechanically forced random wave form, but with two different wind inputs $U_{10}=0$ and $10 \mathrm{~m} \mathrm{~s}^{-1}$, respectively. Figures $3 \mathrm{a}$ and $4 \mathrm{a}$ and Figs. $3 \mathrm{~b}$ and $4 \mathrm{~b}$ are measured at fetch lengths of $x_{\text {wind }} / L=0.65$ and 13.5, respectively. Together these plots visually illustrate some of the well-known effects of wind forcing on the wave heights as the waves propagate downstream. Although identical wave forms are mechanically generated in each case, the resulting surface elevations forced by the wind (shown in Fig. 4) have substantially larger wave heights at $x_{\text {wind }} / L=13.5$ compared to case 1 without wind (Fig. 3). Of particular interest is that a number of the wave groups in case 5 (wind-forced) have very high waves, suggesting increased modulation with wind speed. The statistics and spectral characteristics of the waves and the wave groups are reported hereafter to provide clear evidence of these observations.

\section{a. Development of significant wave height and wave height spectra}

Figure 5a shows the significant wave height as a function of nondimensional fetch length $\left(x_{\text {wind }} / L\right)$ with 
increasing wind speed. Significant wave height $H_{s}$ is calculated as 4 times the standard deviation $\sigma$ of the measured instantaneous wave heights. Case 1 data (circle symbol, $U_{10}=0 \mathrm{~m} \mathrm{~s}^{-1}$ ) indicate that the significant wave height decreases as the waves propagate farther downstream, although the decrease is very weak. This observation is consistent with previous studies (e.g., Onorato et al. 2009) that have observed a loss of wave energy as waves propagate through a finite width tank. However, for the cases with wind input, there is an increase in the significant wave height with fetch length. Miles (1957) classical theory predicts exponential wave growth in time due to the fact that the pressure force from the wind, which is the source of wave growth, increases with the wave height itself. Here we attempt to translate this growth mechanism using a theory for the dependence of stochastic wave growth on wind speed. This is provided as follows. As wind speed increases, a simple energy balance can be formed between lifting a mass of water against gravity and the work done by the lift force of the wind on the water. The lift force (per unit span) is assumed from classical dimensional analysis for an aerodynamic bluff body as

$$
F_{L}=0.5 \rho_{\text {air }} U^{2} H C_{L},
$$

where $H$ is the height of the wave and $C_{L}$ is the lift coefficient. This is essentially a statement that the relevant length scale to the aerodynamic force is the wave height $H$. We will assume $C_{L}$ is approximately constant for the range of wind speeds considered here; breaking waves may have a higher $C_{L}$ (Banner and Melville 1976). There is little basis for this assumption given the lack of knowledge on the details of pressure fields around waves needed to calculate lift, nevertheless we proceed in the pursuit of an outcome that will ultimately be verified by the data. Further, if one ignores wave dynamics under the assumption that the energy transfer from the wind to wave dominates over energy exchange between waves in a wave group, then the wavelength during wave growth is nominally unchanging. This assumption will be further elaborated below when we discuss the spectral characteristics of the waves. Thus, for a small increase in wind speed $d U$ the energy (work) of a small increase in wave height $d H$ due to a small change to the lift force $d F_{L}$ is balanced against the energy (potential) required to lift the small mass of water $d m$ (due to the increase in wave height) against gravity. This analysis leads to

$$
\frac{d H}{d U} \propto H U
$$
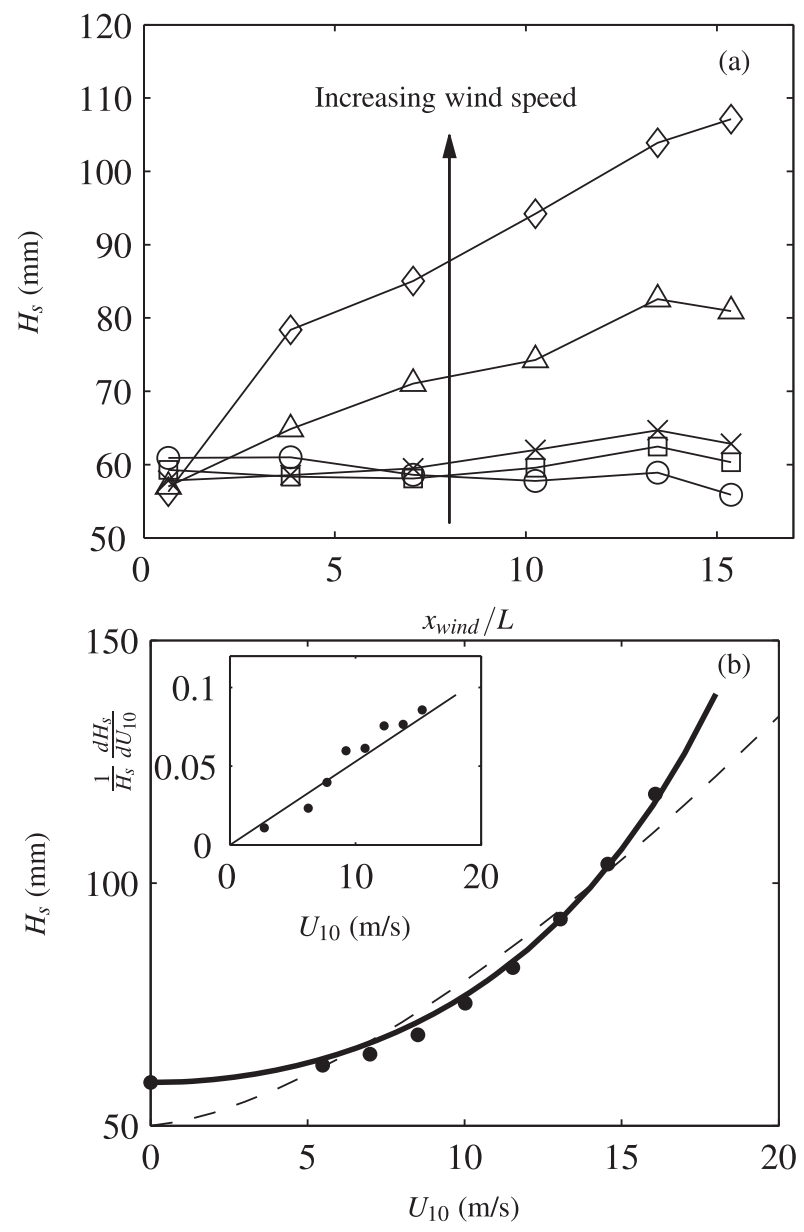

FIG. 5. (a) Significant wave height $H_{s}$ as a function of fetch length with different wind inputs: $U_{10}\left(\mathrm{~m} \mathrm{~s}^{-1}\right) \approx 0(\bigcirc), 5.5(\square), 7.0(\times)$, $11.5(\triangle)$, and $14.6(\diamond)$. (b) $H_{s}$ as a function of wind speeds at $x_{\text {wind }} / L=13.5$ for all the cases listed in Table 1 . The solid line shows the wave growth rate computed using Eq. (5). The dashed line shows $H_{s} \propto\left(U_{10}^{2} x\right)^{0.75}$ as given by Lamont-Smith and Waseda (2008).

with the added assumption that the coefficient of lift $C_{L} \geq O(1)$, which should be a safe assumption for a pressure driven lift force. The data shown in the inset of Fig. 5b show support for Eq. (4). As has been shown by other authors (e.g., Miles 1957), Eq. (4) states that the growth rate of a wave depends on its height, such that integrating this equation leads to the prediction of exponential growth; in this analysis, the growth will be exponential with the square of wind speed,

$$
H_{s}=H_{s 0} \exp \left(A_{1} \frac{1}{2} U^{2}\right)
$$

where $A_{1}$ must be fetch dependent and also a function of wavelength, given that the waves are still developing to reach a stationary state (Toffoli et al. 2017). The term $H_{s 0}$ is the significant wave height at $U_{10}=0$. 

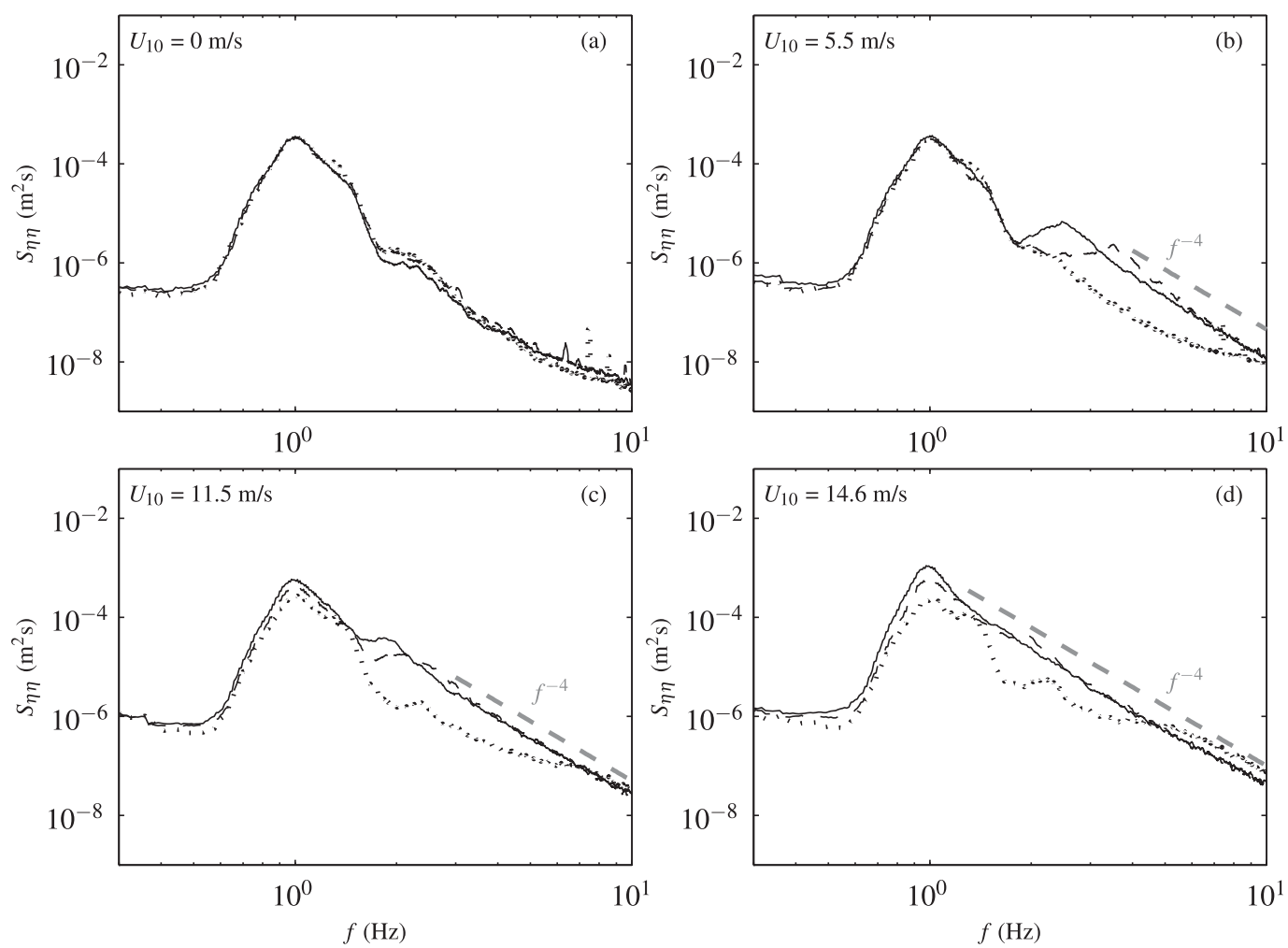

FIG. 6. Evolution of wave spectra with various wind inputs as a function of fetch length: $x_{\text {wind }} / L$ of 0.65 (dotted line), 7.1 (dashed line), and 13.5 (solid line). Gray dashed lines show a power law $f^{-4}$.

Equation (5) is plotted over the data in Fig. 5 and fits well for the range of wind speeds studied (providing evidence that $C_{L}$ may be a constant or, at most, a very weak function of wind speed). Obviously this growth must saturate as wind speed increases, but our data indicate this only occurs at very high wind speeds $\left(U_{10}>16 \mathrm{~m} \mathrm{~s}^{-1}\right)$. The growth is also likely to change for great fetches where a downshift of the dominant frequency waves will eventually occur.

The theoretical growth of $H_{s}$ with wind speed proposed in Eq. (5) is quite different to the data-driven power law $H_{s} \propto\left(U_{10}^{2} x\right)^{0.75}$ proposed in Lamont-Smith and Waseda (2008), which has been shown to describe a range of wind-forced wave data from the laboratory to the field. At higher wind speeds $\left(U_{10} \geq 8 \mathrm{~m} \mathrm{~s}^{-1}\right)$ this power law can be made to agree reasonably with the data acquired here, though it requires an offset to account for the nonzero wave height at zero velocity. Even so, the power law does not describe the full range of wind speeds for these experiments. It is reminded that the experiments conducted here are a very specific, highly controlled case. They serve to clarify the role of wind on waves in the absence of strong wave-wave interactions, specifically, in the absence of downshifting of the dominant waves or focusing/defocusing of wave groups.
Figure 6 shows the evolution of the wave height spectrum at three different fetch lengths and four different wind speeds. For all cases shown in Fig. 6, the peak frequency $f_{0}$ remains nominally constant at $1 \mathrm{~Hz}$, indicating the existing waves (mechanically generated) remain dominant up to a wind fetch of at least $\sim 15$ wavelengths, even at high wind speeds. For the random waves without wind, Fig. 6 a shows the wave height spectra do not change noticeably with fetch. ${ }^{1}$ Figure $6 \mathrm{~b}$ shows the effect of relatively weak wind, where the wind clearly amplifies the higher frequency wave energy. A second peak initially emerges at a frequency approximately 4 times the peak frequency and downshifts as fetch increases (Mitsuyasu and Rikiishi 1978). This peak corresponds to small amplitude, purely wind-generated (short) waves riding over the mechanically generated waves. They grow and downshift quickly with fetch, as pure wind-generated waves do. For the higher wind speed cases shown in

\footnotetext{
${ }^{1}$ As shown in Fig. 5, the significant wave height decreases marginally with fetch for case 1 ; this information is in the spectra, but is spread over a range of wavenumbers such that the effect is too small to observe in Fig. 6a.
} 
Figs. $6 \mathrm{c}$ and $6 \mathrm{~d}$, the secondary peak occurs at a frequency closer to $f_{0}$ for $U_{10}=11.5 \mathrm{~m} \mathrm{~s}^{-1}$ and is completely absorbed into the primary peak for all fetches at $U_{10}=14.6 \mathrm{~m} \mathrm{~s}^{-1}$. Notice that the secondary peak downshift is accompanied by overall growth in the energy of frequencies higher than the secondary peak. This saturates with fetch at given wind speed but continues to grow with wind speed in response to increased amplitude of the pressure fluctuations from the wind field (as shown more clearly in Fig. 8). The observation that the dominant peak does not noticeably shift down in frequency with fetch is a curious one and suggests that the action of the wind is to grow the height of existing waves, but not their wavelength, for a significant fetch (at least 15 wavelengths). Recall that this was a necessary condition to arrive at the exponential growth Eq. (5).

The dashed lines in Fig. 6 represent a power law $f^{-4}$ predicted and observed in the literature (Zakharov and Filonenko 1967; Donelan et al. 1985; Banner 1990; Toffoli et al. 2017). As fetch increases, the range of frequencies following the $f^{-4}$ decay is increased; this range also extends with wind speed. Thus, this controlled experiment has shown that $f^{-4}$ decay of the highest frequency waves is more likely observed at high wind speeds and great fetch lengths.

For engineering purposes, the probability of large waves occurring is important as such waves can be catastrophic to a system (Dysthe et al. 2008). The probability density function of surface elevation with different wind speeds shown in Fig. 7 is an alternative statistical representation of the wind-forced wave evolution. Only the surface elevation distributions measured at a fetch length of $x_{\text {wind }} / L=13.5$ are shown here for brevity, but similar results are observed for all fetch lengths. The shades of gray in Fig. 7 become increasingly darker as the wind speed $U_{10}$ increases from 0 to $16 \mathrm{~m} \mathrm{~s}^{-1}$. For higher wind speeds, the tail of the distribution is longer both for positive and negative surface elevation. This suggests there is an increasing occurrence of very large waves with increasing wind speed for the range of winds tested here. A closer inspection of Fig. 7 reveals that there is a higher probability of very high wave crests compared with similar depth troughs. This asymmetry increases as the waves become steeper and has been attributed to the second-order wave interaction and the presence of bound waves (Onorato et al. 2009). In the ocean, wave crests are higher and wave troughs are shallower than predicted by linear theory, necessitating the inclusion of higher-order terms in ocean surface models (Toffoli et al. 2008).

\section{b. Wind-wave interaction and wave growth}

Figure 8 shows the wave height spectra at $x_{\text {wind }} / L=$ 13.5 as a function of wind speed. Again, the data are

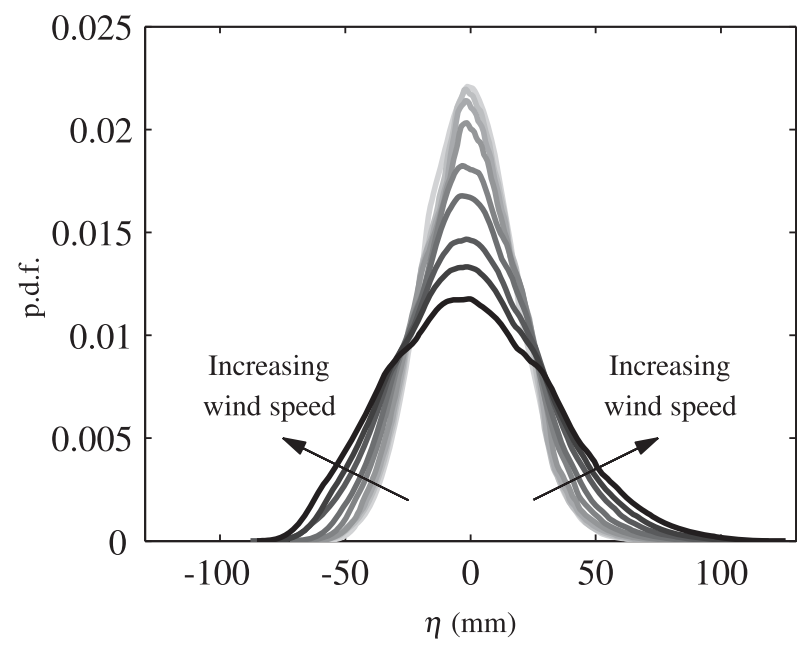

FIG. 7. Probability density function of the surface elevations for different wind speeds at $x_{\text {wind }} / L=13.5$. Increasingly darker shades of gray are shown as $U_{10}$ increases from 0 to $16 \mathrm{~m} \mathrm{~s}^{-1}$.

plotted in progressively darker shades of gray as $U_{10}$ increases. There is a broadband energy increase as the wind speed increases: at the higher frequency range more violent small-scale pressure fluctuations create more short wavelength waves as the wind gets stronger; whereas the larger (low frequency around $f_{0}$ ) waves increase in amplitude due to greater large-scale pressure difference between the windward and leeward faces of the waves (i.e., higher pressure drag) at higher wind speeds. A similar trend in the wave energy spectrum of the Peregrine breather model with wind forcing has been observed by Chabchoub et al. (2013). The inset in Fig. 8 shows a zoomed view of the wave height spectra normalized by the maximum value of the spectra for each case. The normalization is used to highlight changes in the shape of the spectra independently of the increase in peak energy. The width of the spectra for frequencies near $f_{0}$ slightly decreases with the wind speed, that is, there is a subtle narrowing of the spectral peak due to the wind forcing. Janssen (2003) showed the ratio between the wave steepness and the spectral bandwidth is an indicator of the stability of wave fields and referred to the ratio as the BenjaminFeir index (BFI). The BFI is calculated here using the method of Onorato et al. (2006) and shown in Fig. 9 for all the different cases. When $U_{10}=0 \mathrm{~m} \mathrm{~s}^{-1}$, BFI $<1$ for the random wave fields, indicating the wave spectrum is stable without the wind forcing (Janssen 2003). This is expected as we chose initial wave trains that would not break without the influence of wind at any fetch in EASI. However, when there is wind stress, BFI $>1$ and continues to increase with $U_{10}$. The main reason for the BFI increasing so dramatically is that the wave 


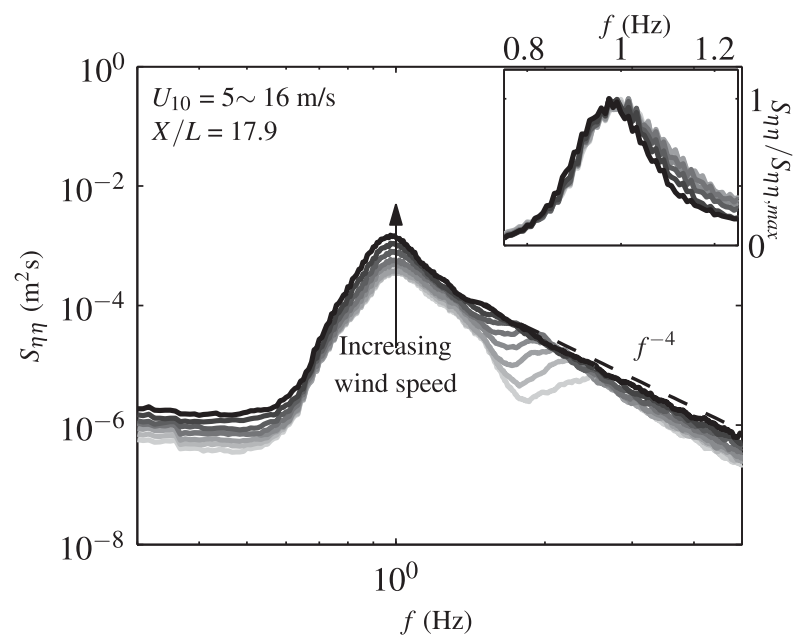

FIG. 8. Evolution of wave spectra as a function of wind speed at $x_{\text {wind }} / L=13.5$. Dashed lines show a power law $f^{-4}$. The inset shows the wave energy spectra normalized by the peak of each spectrum. Increasingly darker shades of gray are shown as $U_{10}$ increases from 0 to $16 \mathrm{~m} \mathrm{~s}^{-1}$.

steepness is increasing with wind speed. The spectral bandwidth is also narrowing with wind speed and this is a secondary reason for the increase. Onorato et al. (2006) showed that BFI increases with increasing $\alpha$ or $\gamma$ since the wave steepness is a function of both JONSWAP spectrum parameters. While $\gamma$ changes the bandwidth of the wave energy spectrum, $\alpha$ is associated with the broadband wave energy. A similar effect is observed with the wind forcing on the existing random sea state wave fields as shown in Fig. 8. This wave energy amplification results in increased wave steepness (because the dominant wavelength is unchanging) and increased BFI. However, the BFI increases with only mild narrowing of the spectral peak, which is what would make the wave fields more unstable, suggesting that the wind-forced waves are not significantly more unstable than the unforced case.

Linear theory predicts the distribution of wave surface elevations, including large waves in random seas, should be well predicted by Gaussian statistics. However since oceanic waves and their interaction are nonlinear, a number of previous studies have observed significant deviations from the Gaussian form and used higher-order statistics to determine the dominant nonlinear wave mechanisms and their effect, especially for mechanically generated waves with directionality and wave fields with a range of different BFI values (Janssen 2003; Toffoli et al. 2008; Onorato et al. 2009; Fedele et al. 2016). For example, the skewness represents the vertical asymmetry of waves associated with the effect of second-order nonlinearities and the presence of bound waves, indicating wave crests

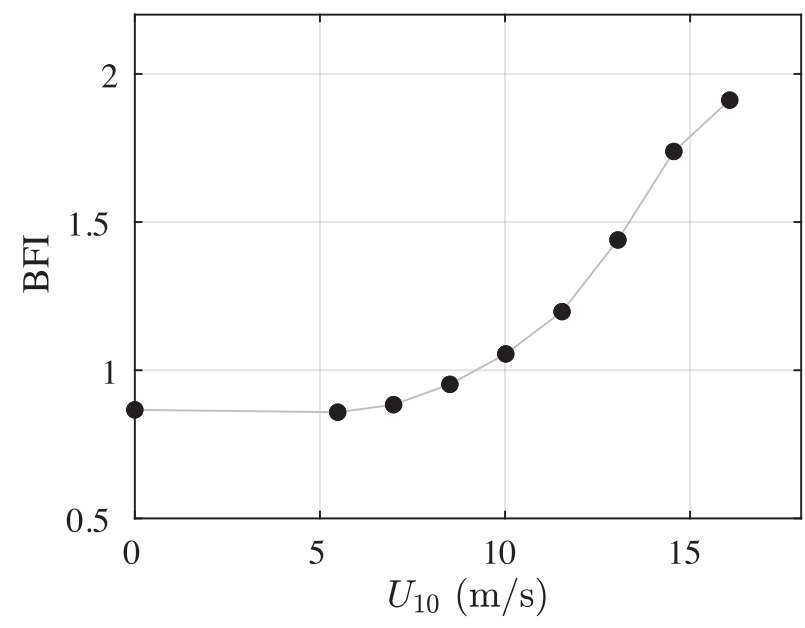

FIG. 9. Benjamin-Feir index (BFI) as a function of wind speeds at $x_{\text {wind }} / L=13.5$.

become sharper whereas wave troughs become shallower with wave steepness (Fedele and Tayfun 2009). On the other hand, the deviation in kurtosis from the Gaussian form indicates the occurrence of extreme waves related to the modulational instability, which is a third-order quasi-resonant process (Janssen 2003). Such nonlinear interaction enhances both wave crests and troughs symmetrically, leading to a substantial deviation in the wave surface distribution beyond the expected second-order predictions (Mori and Janssen 2006). Figures 10a and 10b show skewness and kurtosis of the wave fields as a function of wind speed at three different downstream fetch distances. Figure 10a shows that the random sea state waves without any wind forcing have higher skewness value compared to the Gaussian form (skewness of 0). Interestingly, however, when the wind stress is introduced, the skewness of the wave fields is initially decreased up to $U_{10} \approx 10 \mathrm{~m} \mathrm{~s}^{-1}$ and then increases with increasing wind speeds (for the $x_{\text {wind }} / L$ $\approx 13.5$ the skewness starts increasing at $U_{10} \approx 5 \mathrm{~m} \mathrm{~s}^{-1}$ ). This indicates that initially weak wind forcing suppresses the asymmetry between the wave crests and troughs, but then enhances the asymmetry as the wind forcing gets stronger. Figure 10b displays the kurtosis of the wave fields subjected to an increase in wind forcing. The kurtosis monotonically decreases with wind speed toward the Gaussian value (kurtosis of 3), indicating that the third-order modulational instability weakly decreases with increasing wind forcing. This is a somewhat surprising result given that the BFI shown in Fig. 9 increases with the wind speed. Previously, Toffoli et al. (2008) and Onorato et al. (2009) showed that the kurtosis of measured wave fields is monotonically increased and substantially diverged from the Gaussian process with BFI for long-crested unidirectional waves. 

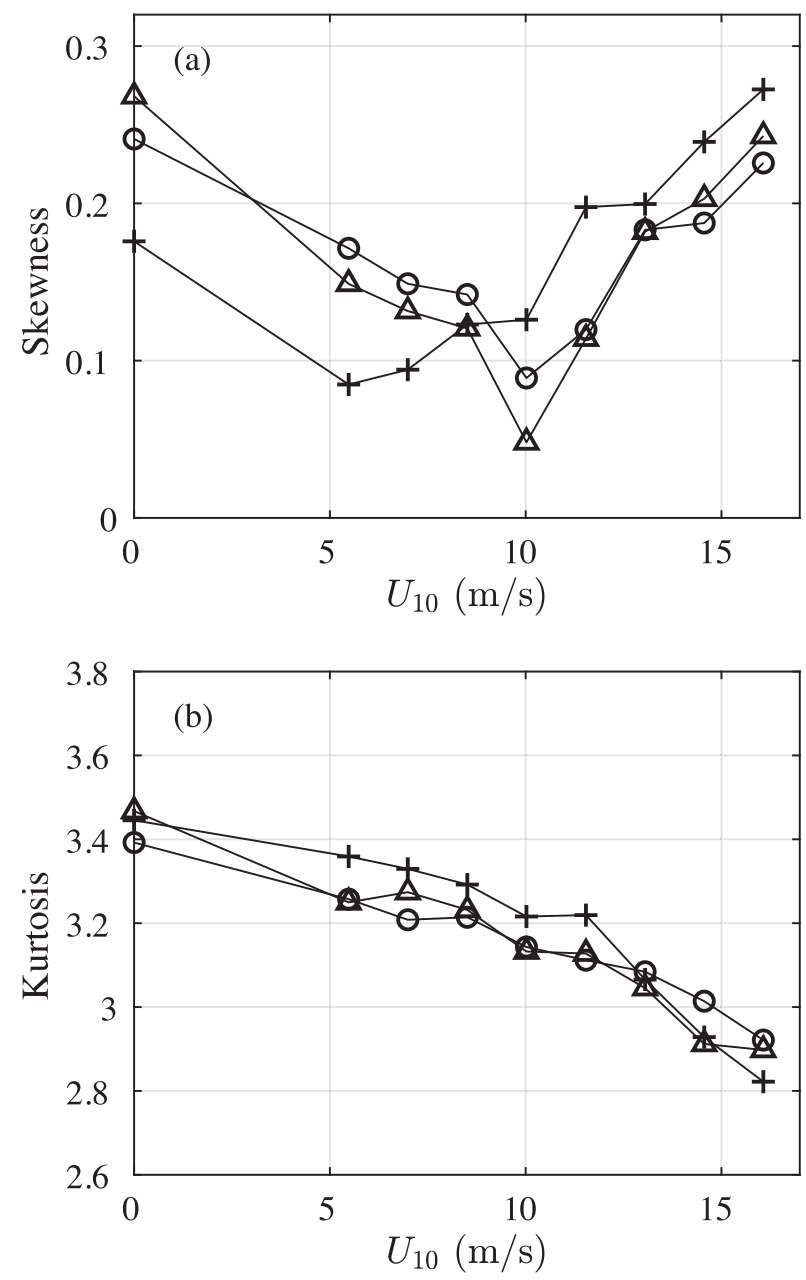

FIG. 10. (a) Skewness and (b) kurtosis of the surface elevation as a function of wind speeds at various fetch lengths: $x_{\text {wind }} / L \approx 3.85$ $(\bigcirc), 7.1(\triangle)$, and $13.5(+)$.

This suggests that the presence of strong wind stress and associated wind-wave interaction dominates the wave dynamics and enhances the wave energy across all the wavelength (which increases BFI) but gradually reduces high-order nonlinear wave dynamics or this decrease of the kurtosis with increasing wind speed could be an artifact of broadband wave growth due to the wind forcing. Before drawing any conclusions about this observation, we seek further evidence for the effect of wind stress on the nonlinear wave interaction in individual wave groups.

First, a selected time series of wave height is chosen for a range of wind speeds and plotted in Fig. 11. The time series is selected such that the largest waves in the wave groups occur at approximately $8 \mathrm{~s}$. It is reminded that the wave trains in this experiment all have the same underlying mechanically generated wave fields, only that they have developed under different wind speeds.

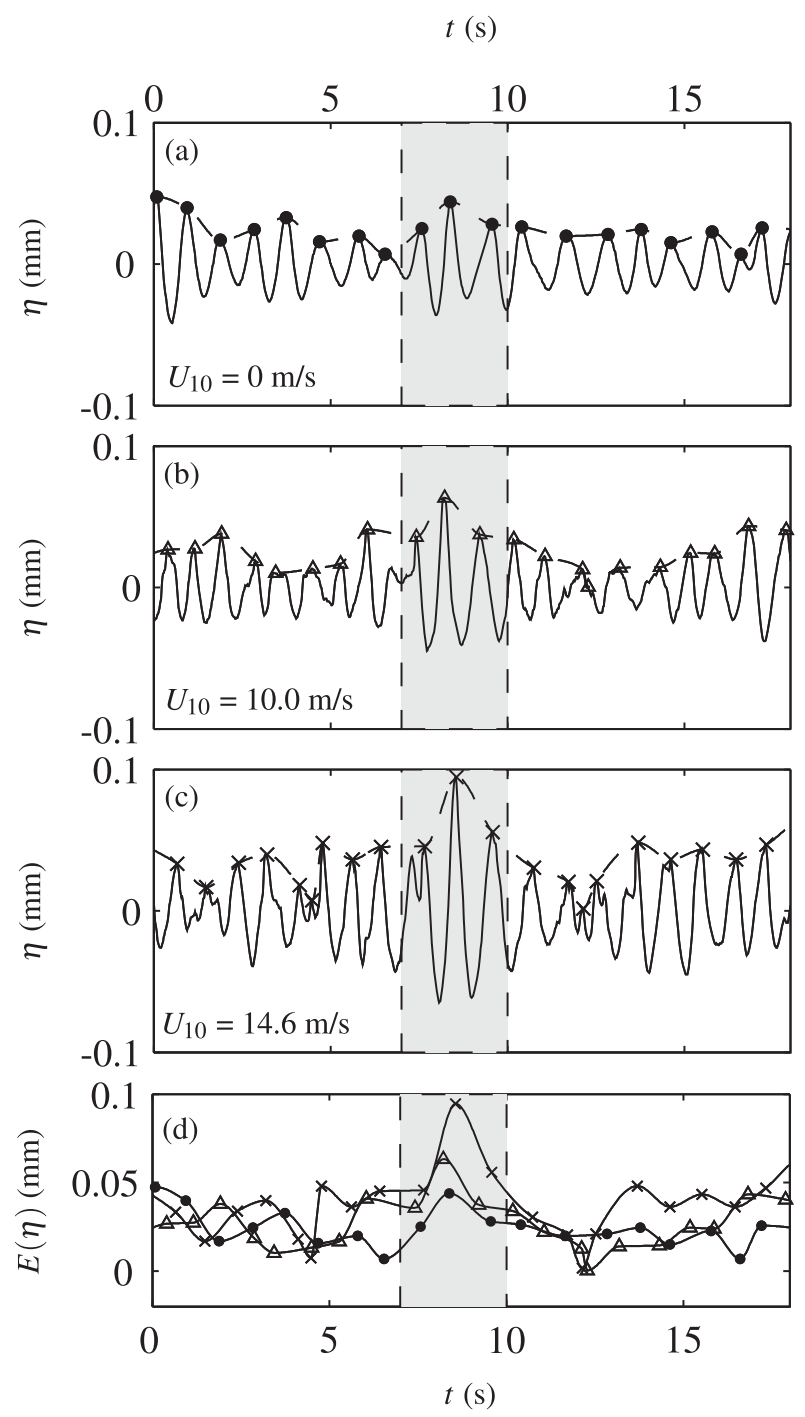

FIG. 11. Example of wave trains with various wind speeds at $x_{\text {wind }} / L=13.5: U_{10}=$ (a) $0.0 \mathrm{~m} \mathrm{~s}^{-1}(\bullet)$, (b) $10.0 \mathrm{~m} \mathrm{~s}^{-1}(\triangle)$, and (c) $14.6 \mathrm{~m} \mathrm{~s}^{-1}(\times)$; the dashed lines with symbols indicate corresponding envelops. (d) Comparison of the magnitude of wave envelops for different wind speeds.

Modulated or enhanced wave groups are clearly evident for all $U_{10}$; however, the envelopes of the wave fields reveal the intensification of the wave growth for the peak wave crest at higher wind speed. In Fig. 11, the dashed lines with symbols indicate the envelope calculated using the Hilbert transform. The detected envelopes for each of the wind speeds are replotted in Fig. $11 \mathrm{~d}$ on the same time scale, where it is more clearly observed that the growth of the peak wave crest is enhanced (highlighted by gray shading in Fig. 11d) relative to the rest of the wave train as $U_{10}$ increases. This is perhaps unsurprising as the largest waves also experience the greatest pressure force, which increases 


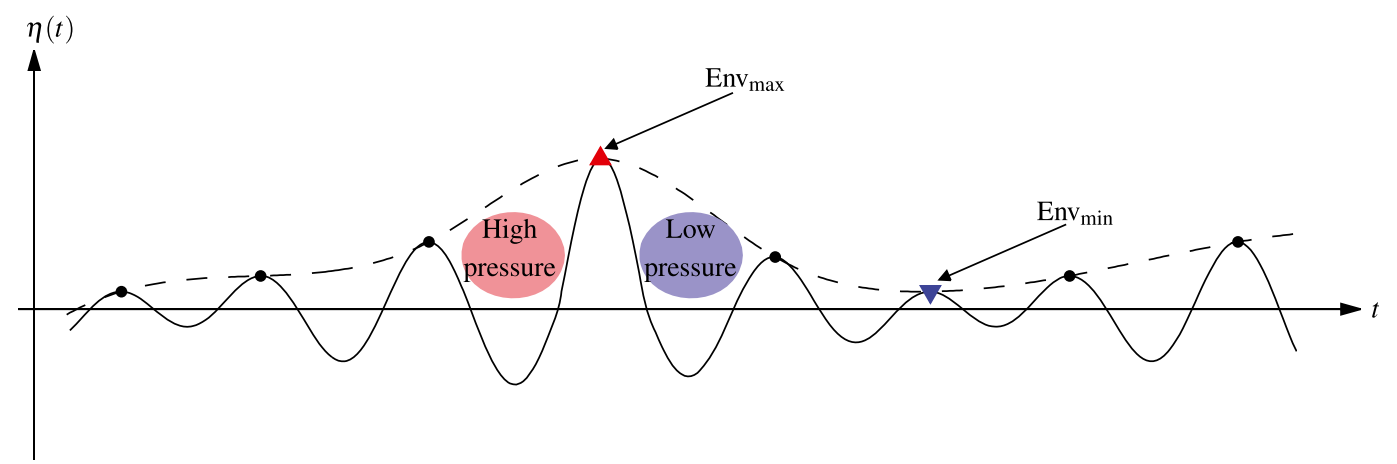

FIG. 12. Schematic of time series of surface elevation measured at a fixed point and its envelope.

their growth faster than smaller waves (Miles 1957) as discussed above.

We now consider all wave groups identified as follows. Figure 12 shows a short, randomly selected time series of a modulated wave group, with the calculated envelope marked with a dashed line. Hereafter, the highest wave crest and the lowest wave crest in the wave group will be referred to as $\mathrm{Env}_{\max }$ and $\mathrm{Env}_{\min }$, respectively. (Their locations are marked by the $\boldsymbol{\Delta}$ and $\boldsymbol{\nabla}$ symbols in the plot). The Hilbert transform is applied to all measured surface elevation datasets $\eta(t)$ to determine the envelope of the wave groups. From the detected envelopes, the values of $E n v_{\text {max }}$ and $E n v_{\text {min }}$ for each wave group can be obtained and we create a database of individual wave groups defined from successive $E_{n v} v_{\text {min }}$. The average of Env $v_{\text {max }}$ and $E_{n v_{\text {min }}}$ over all groups for each wind speed is shown in the inset of Fig. 13. The mean values for both $\operatorname{Env}_{\text {max }}(\boldsymbol{\Delta})$ and $\operatorname{Env}_{\text {min }}(\boldsymbol{\nabla})$ increase with wind forcing, although clearly at different rates. This difference in growth rates means that the highest crest in a wave group grows faster than the lowest crest wave, that is, the wave groups are more modulated in terms of their amplitude, as wind speed increases. Again this is due to the fact that large waves grow faster (with wind speed) than small waves.

Calculating the height difference $H_{\mathrm{env}}=\overline{\mathrm{Env}}_{\max }-\overline{\mathrm{Env}}_{\min }$ between the highest and the lowest crests in wave groups affords a clear view of the wind effects on the modulation characteristics (Fig. 13). The increase in wave amplitude modulation due to the wind is consistent with the aforementioned BFI trend (in Fig. 9), which Janssen (2003) linked to increased susceptibility to third-order quasi-resonant wave interaction associated with modulational instability. Now, although the waves are more modulated by the Miles positive feedback mechanism, the quotient of Env $v_{\text {max }}$ and $E n v_{\text {min }}$ is roughly constant as wind speed increases because both these quantities are growing with wind speed in proportion to their initial height. This is inconsistent with the nonlinear dynamics of free waves occurring in individual groups in the random sea state because this high-order nonlinearity mechanism causes increasing energy in the sidebands, which would manifest as a higher ratio of Env $_{\max }$ to Env $_{\text {min }}$. Indeed all attempts to find even weak evidence of modulation instability occurring in individual wave groups failed, including analyzing the spectra of individual groups (isolated as described above), separating out groups with larger waves and analyzing the fetch development of wave groups. That the BFI is very high and no evidence of modulation instability could be found strongly suggests that in a broadband wave field, the wind does not enhance high-order, nonlinear quasi-resonant wave interactions. This is because the growth of the small waves in a group due to wind force outweighs the attempts of the small waves to transfer energy to the largest wave in the group, as would naturally occur due to modulation instability in the absence of wind. Note that

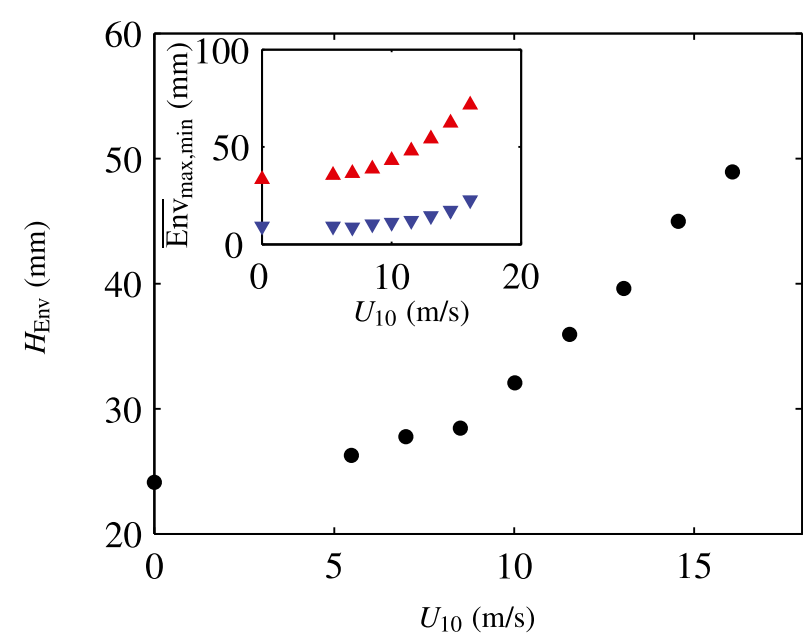

FIG. 13. Modulation height $H_{\text {Env }}$ as a function of wind speed. The inset shows the growth of mean $\operatorname{Env}_{\text {max }}(\boldsymbol{\Delta})$ and $\operatorname{Env}_{\text {min }}(\boldsymbol{\nabla})$ with wind speed. 


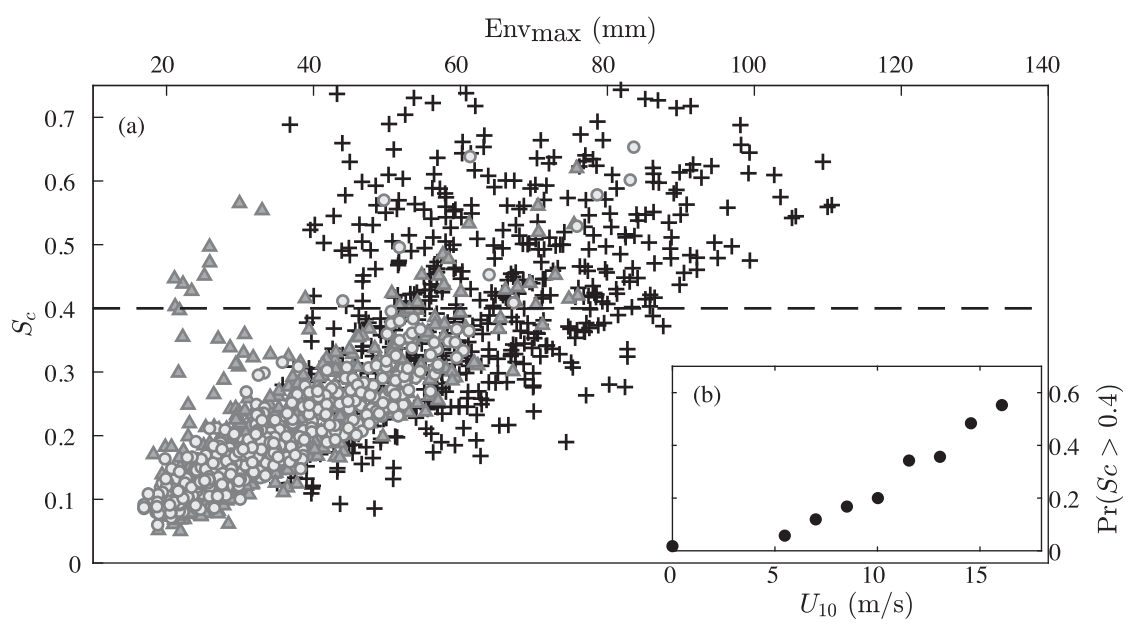

FIG. 14. (a)Local steepness $S_{c}$ of the highest $\left(\operatorname{Env}_{\max }\right)$ waves in the wave group as a function of wind speed: $U_{10}\left(\mathrm{~m} \mathrm{~s}^{-1}\right) \approx 0(\bullet), 5.5(\mathbf{\Delta})$, and $14.5(+)$. (b) Ratio of waves with $S_{c}>0.4$ of the highest $\left(\operatorname{Env}_{\max }\right)$ waves in the wave group as a function of wind speed.

this conclusion can only be drawn for conditions bearing resemblance to those of this controlled experiment, namely, a random sea state subjected to a change in wind speed with relatively young waves and strong wind forcing.

An interesting observation from all the analysis performed so far is that wave breaking does not seem to have marked effects on statistics related to the wave growth, despite visual inspection showing little breaking at the lower wind speeds and more frequent breaking at the highest. For example, breaking might have been expected to affect the wave growth as the analysis above leading to exponential growth was dependent on a constant coefficient of lift, $C_{L}$. Following the findings of Banner and Melville (1976) that airflow separation occurs off breaking waves and this dramatically increases wave drag, it might be expected (though it is not necessary) that the coefficient of lift will also change dramatically following breaking, in line with the Jeffreys sheltering mechanism (Jeffreys 1925). Further, if the largest waves in a group are breaking, this would be expected to reduce the amplitude modulation as the largest waves in a group will break first. Consideration of breaking is problematic due to the well-known difficulty in identifying breaking from temporal wave height records. Recently, Barthelemy et al. (2018) found a strong indicator of breaking was $B_{x}=u_{x} / c_{x}>0.85$, where $B_{x}$ is breaking onset threshold, $u_{x}$ is fluid speed, and $c_{x}$ is wave crest speed in the wave propagation direction, respectively. They also discussed that the local crest steepness $S_{c}=\pi a / \lambda_{c}>0.4$ could be an indicator of breaking in conjunction with $B_{x}$. Here, $a$ is the crest amplitude (which is equivalent to Env $v_{\max }$ of Fig. 14a) and $\lambda_{c}$ is the width of the crest between zero crossings (in the current analysis, the time span of the crest zero crossing period $T_{c}$ is converted to corresponding $\lambda_{c}$ using the dispersion relation). Figure 14a shows the local crest steepness $S_{c}$ for $\mathrm{Env}_{\max }$ for different wind speeds. Without wind, the largest waves almost all have $S_{c}<0.4$, as expected. But as the wind speed is increased from $U_{10}=0$ to $14.5 \mathrm{~m} \mathrm{~s}^{-1}$, the local crest steepness for nnv $_{\text {max }}$ increases and at the highest speed $S_{c}>0.4$ is a common occurrence. In Fig. 14b, the probability of the largest waves in a group having $S_{c}>0.4$ shows a roughly linear increase with wind speed. This is consistent with the observation reported by Onorato et al. (2006) that wave breaking was more frequently observed at larger wave steepness and we previously showed an increasing wave steepness with wind speed (Table 1). Though $S_{c}>0.4$ does not guarantee wave breaking, it does indicate the potential for waves to break in the presence of wind. Therefore, Fig. 14 suggests that at high wind speeds under the conditions studied, large waves in a group are likely to break, while the smaller waves will not, yet this did not appear to significantly affect the statistics of growth.

Somewhat surprisingly, all results shown so far suggest that in realistic sea states, intermittent wave breaking will not be obvious in wave energy spectra or other mean statistics and does not affect the wave growth or nonlinear wave dynamics much, at least not at the range of wind speeds and fetches studied here. This is probably because the majority of waves do not break and not even all of the largest waves in a group necessarily break (Fig. 14 shows a limited probability of breaking for all wind speeds). But it is logical to suggest that as the waves continue to grow with fetch/wind speed, the probability of breaking will increase and this will eventually limit the wave modulation as shown in a relevant study (but 
with very different wave conditions) by Kharif et al. 2008. In that study, an extreme wave event was formed using dispersive focusing with a short frequency modulated wave packet (chirped wave packets) and wind forcing was systematically imposed to investigate the development of focusing and defocusing stages of the extreme wave. Nevertheless, in this random wave field study, wave breaking definitely occurs at higher wind speeds and this undoubtedly limits the growth of the very largest waves (Donelan and Magnusson 2017); this must be statistically represented in probability distributions as discussed in the following section.

\section{c. Exceedance probability of wave crests with wind stress}

We can obtain a clearer picture of the effects of wave breaking on the growth of the larges waves by investigating the exceedance probability of wave crests, defined as the peak elevation of individual waves with respect to the mean water level. Many predictions for the statistical distribution of the wave crests and heights have been published (Winterstein 1988; Tayfun 1980; Forristall 2000; Arena and Fedele 2002; Tayfun and Fedele 2007; Alkhalidi and Tayfun 2013). One of the widely used prediction schemes is the Tayfun (1980) distribution, which is based on the assumptions of deep water and a narrow-banded wave height spectrum. However, the prediction does not consider directional spreading and a substantial deviation appears when waves are long crested (Toffoli et al. 2008; Onorato et al. 2009). Another commonly used prediction scheme is the second-order distribution introduced in Forristall (2000). By performing a large number of simulations using the JONSWAP spectrum, including unidirectional, directional waves with short- and long-crested waves, Forristall (2000) proposed a Weibull distribution with two parameters for both two- and three-dimensional waves, which is given by

$$
P\left(\eta_{c}>\eta\right)=\exp \left[-\left(\frac{\eta}{\alpha H_{s}}\right)^{\beta}\right],
$$

where $\alpha$ and $\beta$ are constants representing the mean wave steepness and Ursell number and $\eta_{c}$ is wave crest elevation. However, there were some observations concerning the accuracy of these predictions in the literature. Toffoli et al. (2008) who compared Euler simulations of long crested waves with the Forristall distribution reported that there is a deviation between the prediction and the simulation at high wave crest regions. Onorato et al. (2009) also observed similar deviations for long-crested waves in comparison with the Tayfun distribution. The experiment in Latheef and
Swan (2013) considered random waves in a number of realistic deep-water sea states with both unidirectional and directionally broad conditions for mechanically generated waves. They reported that while the majority of the measurements were in agreement with the Forristall distribution, there are systematic departures from the models with wave steepness. They also highlighted the importance of directional spreading, indicating the maximum nonbreaking crest height increases with the wave directionality. The change in the exceedance probabilities of high wave crests has also been investigated in previous studies. Onorato et al. (2006) and Shemer et al. (2010) observed that changes in the width of wave spectrum affect the occurrence of large waves, while Toffoli et al. (2008) and Onorato et al. (2009) found similar results when there was a broadbanded directionality in waves. More recently, Alkhalidi and Tayfun (2013) have introduced and generalized the Boccotti distribution to account for the effect of higher-order nonlinearities, especially for relatively large waves. The observed deviation between the original Boccotti's asymptotic distribution (Boccotti 1989) and oceanic datasets is significantly improved after including wave height corrections due to third-order free and bound waves, considering oceanic conditions with wideband directional waves. However, the effect of wind was not considered in any previous studies.

Figure 15 shows the resulting exceedance probability of the wave crests measured in the current experiments with and without wind at $x_{\text {wind }} / L=13.5$. Comparisons of the exceedance probability between the measurement and the Forristall distribution for $U_{10}=0$ and $16.1 \mathrm{~m} \mathrm{~s}^{-1}$ are shown in Figs. 15a and 15b, respectively. The dashed lines show the predictions computed using the Forristall distribution in Eq. (6) with the parameters $\alpha$ and $\beta$ chosen for unidirectional waves. For the no wind case, good agreement is found between the experimental results and the Forristall distribution until $\eta_{c} / H_{s} \approx 1$, but a discrepancy is observed for the highest wave crests, namely the Forristall distribution underestimates the occurrence of high wave crests. Figure $15 \mathrm{~b}$ also exhibits differences between the exceedance probability for the measurement and the Forristall distribution for high wind speeds, especially above $\eta_{c} / H_{s} \approx 0.75$. The opposite behavior is observed compared to the no wind case as the occurrence of high crest waves is now overestimated by the Forristall distribution.

In order to more clearly elucidate the relationship between the wind effect and the occurrence of high crest waves, the exceedance probabilities of the wave crests for the waves developed under a range of wind speed are shown in Figs. 15c and 15d. Increasingly darker shades of gray are shown as $U_{10}$ increases from 


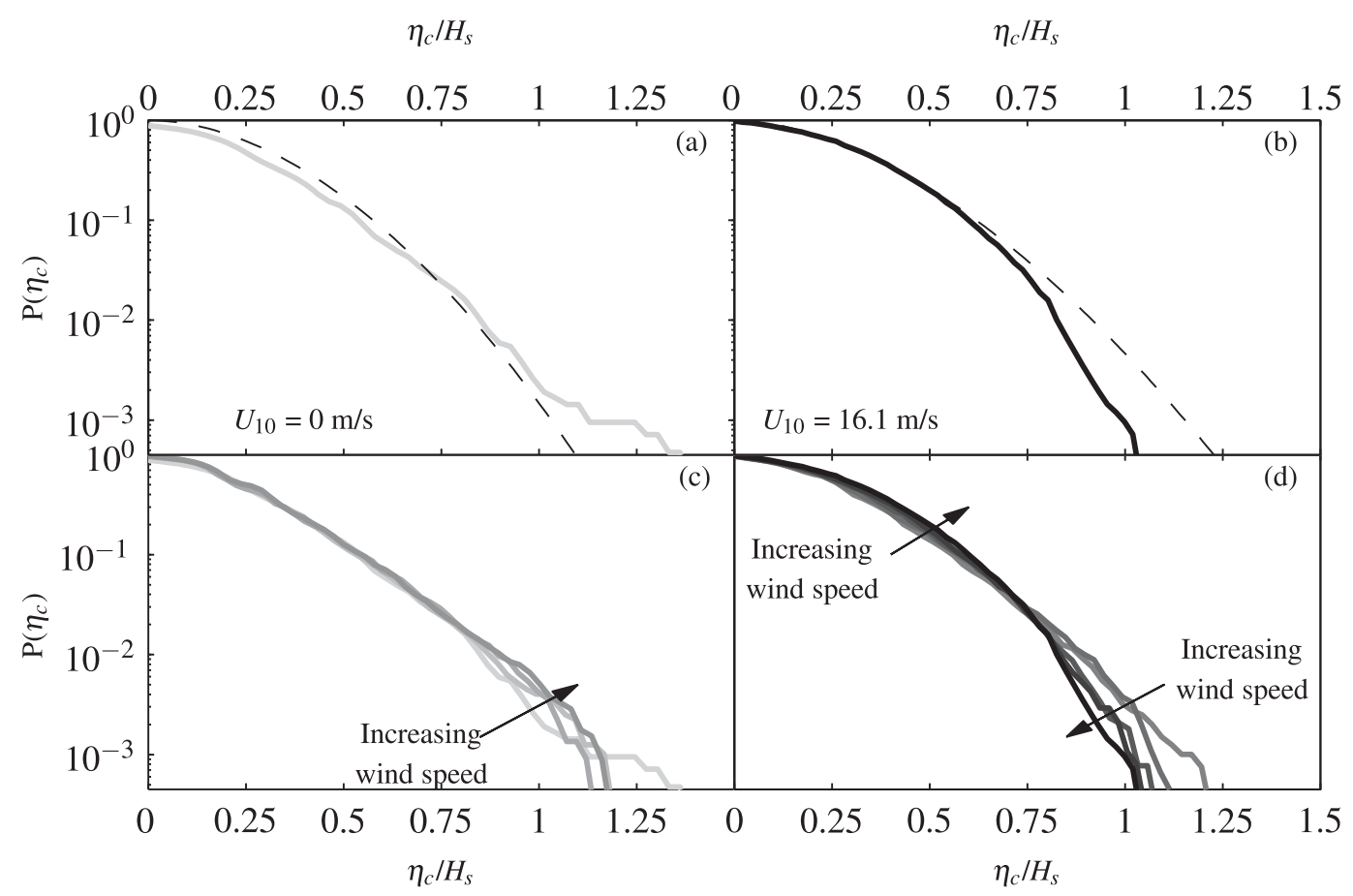

FIG. 15. Exceedance probability of the wave crest $\eta_{c}$ without wind at $x_{\text {wind }} / L=13.5$ compared with second-order simulations of wave crests (Forristall 2000): experimental data (solid line) and the Forristall distributions (dotted line) for unidirectional waves. Increasingly darker shades of gray are shown as $U_{10}$ increases from 0 to $16 \mathrm{~m} \mathrm{~s}^{-1}$.

0 to $16 \mathrm{~m} \mathrm{~s}^{-1}$. For the wind speed range $5.5<U_{10}$ $\left(\mathrm{m} \mathrm{s}^{-1}\right)<10$ (Fig. 15c), the exceedance probability of wave crests larger than $\eta_{c} / H_{s}$ of 0.75 increases with wind speed. However, for the wind speed range $11.5<U_{10}\left(\mathrm{~m} \mathrm{~s}^{-1}\right)<16$ (Fig. 15d), indicates the probability of occurrence of high amplitude waves reduces with increasing wind speed and even drops below the prediction at the probability level $P\left(\eta_{c}\right)=$ 0.01 for the highest wind input $U_{10}=16.1 \mathrm{~m} \mathrm{~s}^{-1}$. Hence, there is a clear increasing and then decreasing behavior in the tail of the exceedance probabilities with the wind. A similar trend is observed by Latheef and Swan (2013) for both unidirectional and directional waves. They attributed the higher crest elevation (compared to the Forristall distribution) to the additional nonlinear amplification beyond second order. However, they found a competing mechanism which suppresses this nonlinear wave growth as the wave steepness increases. For both unidirectional and directional waves, it was observed that this mechanism induced a significant reduction in the exceedance probability of the crest height below the probability level $P\left(\eta_{c}\right)=0.01$ and overestimated by the Forristall distribution at a high wave steepness. They concluded that this limiting mechanism is associated with wave breaking and as supporting evidence, they showed the nonlinear amplification and the onset of wave breaking are larger in the unidirectional waves than the directional waves, which can sustain further wave growth without breaking. For the current study, however, the wave fields with different wind forcing are nominally unidirectional. Therefore, this suggests that deviation from the Forristall distribution, as evident in Fig. 15, is mainly due to the growth of peak wave crests in a wave group, which is due to the energy transfer from the wind, leading to increasing local wave steepness and hence the occurrence of wave breaking (Toffoli et al. 2010). Gemmrich and Farmer (1999) have also reported previously that the frequency of wave breaking is increased with wind energy input, consistent with our results in Figs. 14 and 15. Clearly the extra energy input from the wind into propagating waves plays an important role on the exceedance probability of wave crests and hence the local wind (or associated wave growth rate) should be included in predictions of the statistical properties of ocean waves.

\section{Conclusions}

A laboratory experiment in a large wind-wave facility has been performed to study the effect of wind input on random sea state waves. The experiments were carefully planned and executed to isolate only the effects of wind and associated wind-wave interaction 
on waves of a fixed dominant frequency. Results confirm that additional wind energy enhances the instantaneous surface elevations, increasing the significant wave height with wind speed at a given fetch length as anticipated. When $H_{s}$ is plotted against wind speed, the growth rate is clearly exponential. A preliminary functional form, which describes the wave growth rate with wind speed well, is derived using classical dimensional analysis.

As the wind speed increases, additional wind energy disproportionately affects waves according to their height such that amplitude modulation of the waves is increased with increasing wind speed. This is likely due to relatively higher wind-induced pressure drag on the larger waves and results in the largest waves being pushed to a steepness beyond the breaking limit by the wind. Despite the increase in the wave amplitude modulation, there is no clear evidence of enhancement in high-order nonlinear wave interactions with the additional wind energy. This suggests that when waves develop under the competing effects of wind forcing and nonlinear wave interaction, wind-wave interaction due to wind forcing dominates the growth mechanism over the wave-wave interaction. Note that the results discussed here are relevant to the development of existing random sea state wave fields subjected to a strong wind forcing. However, the sea state in the current study is limited to unidirectional random wave fields with the wind forcing in the direction of wave propagation. Also, although the random sea state waves are developed under the strong wind conditions, the dominant wave fields are still mechanically generated two-dimensional wave fields.

The exceedance probability of wave crests is found to depend on wind speed most obviously for the lowest probability (largest) waves, where a deviation from empirical second-order models is clearly evident. At lower wind speed, the Forristall distribution underestimates the probability of occurrence of the high amplitude waves, yet overpredicts at high wind speeds. This overprediction is mainly because, at the high wind speeds, the growth of extreme waves is limited by the wave breaking mechanism as the local wave steepness increases with wind speed. It is suggested that exceedance probability models include a parameter for wind speed (as a surrogate for breaking of the largest waves).

Following the finding that wave amplitude modulation is increased by wind, further studies need to be conducted with a greater range of parameters including wave age, fetch, and wave directionality. This will provide the necessary data to model the complex physics governing wind effects on wave dynamics, leading to the breaking of large waves.
Acknowledgments. The authors gratefully acknowledge support from Australian Research Council. The authors also wish to thank A. V. Babanin and G. Wake for valuable ideas and discussions and A. Toffoli for providing the wave gauges.

\section{REFERENCES}

Alkhalidi, M. A., and M. A. Tayfun, 2013: Generalized Boccotti distribution for nonlinear wave heights. Ocean Eng., 74, 101106, https://doi.org/10.1016/j.oceaneng.2013.09.014.

Arena, F., and F. Fedele, 2002: A family of narrow-band non-linear stochastic processes for the mechanics of sea waves. Eur. J. Mech., 21B, 125-137, https://doi.org/10.1016/S0997-7546(01)01167-0.

Banner, M. L., 1990: Equilibrium spectra of wind waves. J. Phys. Oceanogr., 20, 966-984, https://doi.org/10.1175/1520-0485(1990) 020<0966:ESOWW >2.0.CO;2.

Banner, M. I., and W. K. Melville, 1976: On the separation of air flow over water waves. J. Fluid Mech., 77, 825-842, https:// doi.org/10.1017/S0022112076002905.

Barthelemy, X., M. I. Banner, W. L. Peirson, F. Fedele, M. Allis, and F. Dias, 2018: On a unified breaking onset threshold for gravity waves in deep and intermediate depth water. J. Fluid Mech., 841, 463-488, https://doi.org/10.1017/jfm.2018.93.

Benjamin, T. B., and J. E. Feir, 1967: The disintegration of wave trains on deep water: Part 1. Theory. J. Fluid Mech., 27, 417430, https://doi.org/10.1017/S002211206700045X.

Bliven, L. F., N. E. Huang, and S. R. Long, 1986: Experimental study of the influence of wind on Benjamin-Feir sideband instability. J. Fluid Mech., 162, 237-260, https://doi.org/10.1017/ S0022112086002033.

Boccotti, P., 1989: On mechanics of irregular gravity waves. Atti Accad. Naz. Lincei Mem., 19, 111-170.

Brunetti, M., and J. Kasparian, 2014: Modulational instability in wind-forced waves. Phys. Lett. A, 378, 3626-3630, https:// doi.org/10.1016/j.physleta.2014.10.017.

Chabchoub, A., N. P. Hoffmann, and N. Akhmediev, 2011: Rogue wave observation in a water wave tank. Phys. Rev. Lett., 106, 204502, https://doi.org/10.1103/physrevlett.106.204502.

_- N. Hoffmann, H. Branger, C. Kharif, and N. Akhmedievl, 2013: Experiments on wind-perturbed rogue wave hydrodynamics using the Peregrine breather model. Phys. Fluids, $\mathbf{2 5}$, 101704, https://doi.org/10.1063/1.4824706.

Denissenko, P., S. Lukaschuk, and S. Nazarenko, 2007: Gravity wave turbulence in a laboratory flume. Phys. Rev. Lett., 99, 014501, https://doi.org/10.1103/physrevlett.99.014501.

Donelan, M. A., and A. K. Magnusson, 2017: The making of the Andrea wave and other rogues. Sci. Rep., 7, 44124, https:// doi.org/10.1038/srep44124.

_ J. Hamilton, and W. H. Hui, 1985: Directional spectra of wind-generated waves. Philos. Trans. Roy. Soc. London, 315A, 509-562, https://doi.org/10.1098/rsta.1985.0054.

Dysthe, K., K. Trulsen, H. E. Krogstad, and H. Socquet-Juglard, 2003: Evolution of a narrow-band spectrum of random surface gravity waves. J. Fluid Mech., 478, 1-10, https://doi.org/ 10.1017/S0022112002002616.

- H. E. Krogstad, and P. Müller, 2008: Oceanic rogue waves. Annu. Rev. Fluid Mech., 40, 287-310, https://doi.org/10.1146/ annurev.fluid.40.111406.102203.

Fedele, F., and M. A. Tayfun, 2009: On nonlinear wave groups and crest statistics. J. Fluid Mech., 620, 221-239, https://doi.org/ 10.1017/S0022112008004424. 
J. Brennan, S. P. de León, J. Dudley, and F. Dias, 2016: Real world ocean rogue waves explained without the modulational instability. Sci. Rep., 6, 27715, https://doi.org/10.1038/srep27715.

Forristall, G. Z., 2000: Wave crests distributions: Observations and second-order theory. J. Phys. Oceanogr., 30, 1931-1943, https://doi.org/10.1175/1520-0485(2000)030<1931:WCDOAS > 2.0.CO;2.

Gemmrich, J. R., and D. M. Farmer, 1999: Observations of the scale and occurrence of breaking surface waves. J. Phys. Oceanogr., 29, 2595-2606, https://doi.org/10.1175/1520-0485(1999)029<2595: OOTSAO $>2.0 . \mathrm{CO} ; 2$

Janssen, P. A., 2003: Nonlinear four-wave interactions and freak waves. J. Phys. Oceanogr., 33, 863-884, https://doi.org/10.1175/ 1520-0485(2003)33<863:NFIAFW $>2.0 . \mathrm{CO} ; 2$.

Jeffreys, H., 1925: On the formation of wave by wind. Proc. Roy. Soc. London, 107A, 189-206, https://doi.org/10.1098/ rspa.1925.0015.

Kharif, C., and E. Pelinovsky, 2003: Physical mechanisms of the rogue wave phenomenon. Eur. J. Mech., 22B, 603-634, https:// doi.org/10.1016/j.euromechflu.2003.09.002.

_ , J.-P. Giovanangeli, J. Touboul, L. Grare, and E. Pelinovsky, 2008: Influence of wind on extreme wave events: experimental and numerical approaches. J. Fluid Mech., 594, 209-247, https://doi.org/10.1017/S0022112007009019.

Komen, G. J., L. Cavaleri, and M. Donelan, 1994: Dynamics and Modelling of Ocean Waves. Cambridge University Press, $556 \mathrm{pp}$.

Lamont-Smith, T., and T. Waseda, 2008: Wind wave growth at short fetch. J. Phys. Oceanogr., 38, 1597-1606, https://doi.org/ 10.1175/2007JPO3712.1.

Latheef, M., and C. Swan, 2013: A laboratory study of wave crest statistics and the role of directional spreading. Proc. Roy. Soc. London, 469A, 20120696, https://doi.org/10.1098/rspa.2012.0696.

Lee, J. H., J. P. Monty, J. Elsnab, A. Toffoli, A. V. Babanin, and A. Alberello, 2017: Estimation of kinetic energy dissipation from breaking waves in the wave crest region. J. Phys. Oceanogr., 47, 1145-1150, https://doi.org/10.1175/JPO-D-160273.1.

Li, J. C., W. H. Hui, and M. A. Donelan, 1988: Effects of velocity shear on the stability of surface deep water wave trains. Nonlinear Water Waves, K. Horikawa and H. Maruo, Springer, 213-220.

Longuet-Higgins, M. S., 1963: The effect of non-linearities on statistical distribution in the theory of sea waves. J. Fluid Mech. 17, 459-480, https://doi.org/10.1017/S0022112063001452.

Miles, J. W., 1957: On the generation of surface waves by shear flows. J. Fluid Mech., 3, 185-204, https://doi.org/10.1017/ S0022112057000567.

Mitsuyasu, H., and K. Rikiishi, 1978: The growth of durationlimited wind waves. J. Fluid Mech., 85, 705-730, https:// doi.org/10.1017/S0022112078000889.

Mori, N., and P. A. Janssen, 2006: On kurtosis and occurrence probability of freak waves. J. Phys. Oceanogr., 36, 1471-1483, https://doi.org/10.1175/JPO2922.1.
Onorato, M. O., A. R. Osborne, M. Serio, L. Cavaleri, C. Brandini, and C. T. Stansberg, 2006: Extreme waves, modulational instability and second order theory: Wave flume experiments on irregular waves. Eur. J. Mech., 25B, 586-601, https://doi.org/10.1016/ j.euromechflu.2006.01.002.

— , and Coauthors, 2009: Statistical properties of mechanically generated surface gravity waves: A laboratory experiment in a three-dimensional wave basin. J. Fluid Mech., 627, 235-257, https://doi.org/10.1017/S002211200900603X.

Shemer, L., A. Sergeeva, and D. Liberzon, 2010: Effect of the initial spectrum on the spatial evolution of statistics of unidirectional nonlinear random waves. J. Geophys. Res., 115, C12039, https://doi.org/10.1029/2010JC006326.

Socquet-Juglard, H., K. Dysthe, K. Trulsen, H. E. Krogstad, and J. Liu, 2005: Probability distributions of surface gravity waves during spectral changes. J. Fluid Mech., 542, 195-216, https:// doi.org/10.1017/S0022112005006312.

Tayfun, M. A., 1980: Narrow-band nonlinear sea waves. J. Geophys. Res., 85, 1548-1552, https://doi.org/10.1029/JC085iC03p01548.

, and F. Fedele, 2007: Wave-height distributions and nonlinear effects. Ocean Eng., 34, 1631-1649, https://doi.org/10.1016/ j.oceaneng.2006.11.006.

Toffoli, A., E. Bitner-Gregersen, M. Onorato, and A. V. Babanin, 2008: Wave crest and trough distributions in a broad-banded directional wave field. Ocean Eng., 35, 1784-1792, https:// doi.org/10.1016/j.oceaneng.2008.08.010.

_ - A. Babanin, M. Onorato, and T. Waseda, 2010: Maximum steepness of oceanic waves: Field and laboratory experiments. Geophys. Res. Lett., 37, L05603, https://doi.org/10.1029/ 2009GL041771.

— , and Coauthors, 2017: Wind generated rogue waves in an annular wave flume. Phys. Rev. Lett., 118, 144503, https:// doi.org/10.1103/physrevlett.118.144503.

Waseda, T., 2006: Impact of directionality on the extreme wave occurrence in a discrete random wave system. Proc. 9th Int. Workshop on Wave Hindcasting and Forecasting, Victoria, BC, Canada, Environment Canada, 10 pp., http:// www.waveworkshop.org/9thWaves/Papers/Waseda.pdf.

, and M. P. Tulin, 1999: Experimental study of the stability of deep-water wave trains including wind effects. J. Fluid Mech., 401, 55-84, https://doi.org/10.1017/S0022112099006527.

White, B. S., and B. Fornberg, 1998: On the chance of freak waves at sea. J. Fluid Mech., 355, 113-138, https://doi.org/10.1017/ S0022112097007751.

Winterstein, S. R., 1988: Nonlinear vibration models for extremes and fatigue. J. Eng. Mech., 114, 1772-1790, https://doi.org/ 10.1061/(ASCE)0733-9399(1988)114:10(1772).

Zakharov, V. E., 1968: Stability of periodic waves of finite amplitude on the surface of a deep fluid. J. Appl. Mech. Tech. Phys., 9, 190-194, https://doi.org/10.1007/BF00913182.

, and N. N. Filonenko, 1967: Energy spectrum for stochastic oscillations of the surface of a liquid. Sov. Phys. Dokl., 11, 881-883. 